\title{
DIFFERENCES OF MEANS
}

BY O. SHISHA AND B. MOND

Communicated by J. B. Diaz, December 12, 1966

1. Let $q_{1}, q_{2}, \cdots, q_{n}$ be positive numbers with $\sum_{k=1}^{n} q_{k}=1$. For every sequence $\left(x_{1}, x_{2}, \cdots, x_{n}\right)$ with all $x_{k}>0$ and for every real $r$, consider the mean of order $r, M_{r}\left(x_{1}, x_{2}, \cdots, x_{n}\right)$, defined as $\left(\sum_{k=1}^{n} q_{k} x_{k}^{r}\right)^{1 / r}$ if $r \neq 0$, and as $\prod_{k=1}^{n} x_{k}^{q_{k}}$ if $r=0$. For given positive $x_{1}, x_{2}, \cdots, x_{n}$, it is known (see, e.g. [3, p. 17], or [11, p. 26]) that $M_{r}\left(x_{1}, x_{2}, \cdots, x_{n}\right)$ is strictly increasing with $r$ (except when $x_{1}$ $\left.=x_{2} \ldots=x_{n}\right)$, and consequently if $r<s$, then

$$
\begin{aligned}
& 1 \leqq M_{s}\left(x_{1}, x_{2}, \cdots, x_{n}\right) / M_{r}\left(x_{1}, x_{2}, \cdots, x_{n}\right), \\
& 0 \leqq M_{s}\left(x_{1}, x_{2}, \cdots, x_{n}\right)-M_{r}\left(x_{1}, x_{2}, \cdots, x_{n}\right) .
\end{aligned}
$$

2. A natural question to ask is, whether one can give upper bounds for the right hand sides of (1) and (2) under, say, the hypothesis that $A \leqq x_{j} \leqq B, j=1,2, \cdots, n$, where $A$ and $B$ are constants $(0<A<B)$. Such an upper bound for the ratio in (1) was given by Cargo and Shisha in [4], a paper which served as a motivation and starting point of a considerable amount of further work by various authors.

3. The main purpose of the present note is to give an upper bound for the difference in (2) under the restriction on the $x_{j}$ stated in $\$ 2$. As applications, we shall obtain a number of inequalities, including "complements" to the classical inequalities of Cauchy and Hölder. Full proofs omitted here are to be found in [15].

4. In this section, $q_{1}, q_{2}, \cdots, q_{n}$ are fixed (though arbitrary) positive numbers with $\sum_{k=1}^{n} q_{k}=1$, and for every sequence $\left(x_{1}, x_{2}, \cdots, x_{n}\right)$ with all $x_{k}>0$ and every real $r, M_{r}\left(x_{1}, x_{2}, \cdots, x_{n}\right)$ is as in $\S 1$.

Theorem 1. Let $r, s, A, B$ be given reals $(0<A<B, r<s)$, and let $I$ denote the $n$-dimensional cube $\left\{\left(x_{1}, x_{2}, \cdots, x_{n}\right): A \leqq x_{k} \leqq B\right.$, $k=1,2, \cdots, n\}$. Then throughout $I$,

$$
M_{s}\left(x_{1}, x_{2}, \cdots, x_{n}\right)-M_{r}\left(x_{1}, x_{2}, \cdots, x_{n}\right) \leqq \Delta,
$$

where $\Delta$ is

$$
\begin{aligned}
& {\left[\theta B^{s}+(1-\theta) A^{s}\right]^{1 / s}-\left[\theta B^{r}+(1-\theta) A^{r}\right]^{1 / r} \quad \text { if } r s \neq 0,} \\
& {\left[\theta B^{s}+(1-\theta) A^{s}\right]^{1 / s}-B^{\theta} A^{1-\theta} \quad \text { if } r=0,}
\end{aligned}
$$

and 


$$
B^{\theta} A^{1-\theta}-\left[\theta B^{r}+(1-\theta) A^{r}\right]^{1 / r} \quad \text { if } s=0 .
$$

$\theta$ is defined as follows. Let

$$
\begin{gathered}
h(x) \equiv x^{1 / s}-(a x+b)^{1 / r} \text { with } a=\frac{B^{r}-A^{r}}{B^{s}-A^{s}} \\
b=\frac{B^{s} A^{r}-B^{r} A^{s}}{B^{s}-A^{s}}, \quad \text { if } r s \neq 0, \\
h(x) \equiv x^{1 / s}-A(B / A)^{\left(x-A^{s}\right) /\left(B^{s}-A^{s}\right)} \quad \text { if } r=0,
\end{gathered}
$$

and

$$
h(x) \equiv-x^{1 / r}+A(B / A)^{(x-A r) /(B r-A r)} \quad \text { if } s=0 .
$$

Let $J$ denote the open interval joining $A^{s}$ to $B^{s}$ if $s \neq 0$, and let $J=\left(B^{r}, A^{r}\right)$ if $s=0$. There is an $x^{*} \in J$ such that $h(x)<h\left(x^{*}\right)$ for every $x\left(\in J, \neq x^{*}\right)$. (Observe that if $r s \neq 0$, then $a x+b>0$ at the end points of $J$ and, therefore, throughout $J$.) We set

and

$$
\theta=\left(x^{*}-A^{s}\right) /\left(B^{s}-A^{s}\right) \quad \text { if } s \neq 0,
$$

$$
\theta=\left(x^{*}-A^{r}\right) /\left(B^{r}-A^{r}\right) \quad \text { if } s=0 .
$$

Equality in (3) for a point $\left(x_{1}, x_{2}, \cdots, x_{n}\right) \in I$ holds if and only if there exists a subsequence $\left(k_{1}, k_{2}, \cdots, k_{p}\right)$ of $(1,2, \cdots, n)$ such that $\sum_{m=1}^{p} q_{k_{m}}=\theta, x_{k_{m}}=B(m=1,2, \cdots, p)$, and $x_{k}=A$ for every $k$ distinct from all $k_{m}$. Finally, if $s \geqq 1$, then $x^{*}$ is the unique solution of $h^{\prime}(x)=0$ in $J$.

5. Here is an outline of a proof of Theorem 1. Suppose $r s \neq 0$. Then [12] throughout $(A, B)$,

$$
r\left(x^{r}-a x^{s}-b\right)>0 .
$$

Let $\left(x_{1}, x_{2}, \cdots, x_{n}\right)$ be a point of $I$. Then

$$
r\left[\left(\sum_{k=1}^{n} q_{k} x_{k}^{r}\right)-a\left(\sum_{k=1}^{n} q_{k} x_{k}^{s}\right)-b\right] \geqq 0,
$$

and so,

$$
\begin{aligned}
M_{s}\left(x_{1}, x_{2}, \cdots, x_{n}\right)-M_{r}\left(x_{1}, x_{2}, \cdots, x_{n}\right) & \leqq \\
& \leqq\left(\sum_{k=1}^{n} q_{k} x_{k}^{8}\right)^{1 / s}-\left[a\left(\sum_{k=1}^{n} q_{k} x_{k}^{s}\right)+b\right]^{1 / r} \\
& =h\left(\sum_{k=1}^{n} q_{k} x_{k}^{8}\right) .
\end{aligned}
$$


One shows that there is a unique point in the closure of $J$ where $h(x)$ attains its maximum there, and this point, $x^{*}$, belongs to $J$. Thus,

$$
\begin{aligned}
M_{s}\left(x_{1}, x_{2}, \cdots, x_{n}\right) & -M_{r}\left(x_{1}, x_{2}, \cdots, x_{n}\right) \\
& \leqq h\left(x^{*}\right) \\
& =h\left(\theta B^{s}+(1-\theta) A^{s}\right) \\
& =\left[\theta B^{s}+(1-\theta) A^{s}\right]^{1 / s}-\left[\theta B^{r}+(1-\theta) A^{r}\right]^{1 / r}=\Delta .
\end{aligned}
$$

In case $s \geqq 1$, one can also prove the theorem by the method used in [4] to obtain an upper bound for the right hand side of (1). Namely, suppose $n>1, r \neq 0$, and set $F\left(x_{1}, x_{2}, \cdots, x_{n}\right) \equiv M_{s}\left(x_{1}, x_{2}, \cdots, x_{n}\right)$ $-M_{r}\left(x_{1}, x_{2}, \cdots, x_{n}\right)$; one shows that a point of $I$ where $F$ attains its maximum in $I$ must be a vertex of $I$. Thus, the last difference is bounded in $I$ by $\max \left\{\left[x B^{s}+(1-x) A^{s}\right]^{1 / s}-\left[x B^{r}+(1-x) A^{r}\right]^{1 / r}\right.$ : $0 \leqq x \leqq 1\}$ which equals $\Delta$.

REMARK. If $r \neq 0, s \geqq 1$, then $\theta$ of Theorem 1 is the unique solution in $(0,1)$ of

$$
\begin{aligned}
& \frac{\gamma^{s}-1}{s}\left[x\left(\gamma^{s}-1\right)+1\right]^{(1 / s)-1}-\frac{\gamma^{r}-1}{r}\left[x\left(\gamma^{r}-1\right)+1\right]^{(1 / r)-1}=0 \\
&(\gamma=B / A) .
\end{aligned}
$$

6. Here are two examples.

EXAMPLE 1. Let $q_{1}, q_{2}, \cdots, q_{n}$ be positive numbers with $\sum_{k=1}^{n} q_{k}=1$, let $0<A<B$, and set $\gamma=B / A$. Let $A \leqq x_{k} \leqq B, k=1,2, \cdots, n$. By Theorem 1 and by the preceding Remark,

$$
\left(\sum_{k=1}^{n} q_{k} x_{k}^{2}\right)^{1 / 2}-\sum_{k=1}^{n} q_{k} x_{k} \leqq\left[\theta B^{2}+(1-\theta) A^{2}\right]^{1 / 2}-[\theta B+(1-\theta) A],
$$

where $\theta$ is the unique solution in $(0,1)$ of

$$
\frac{1}{2}\left(\gamma^{2}-1\right)\left[x\left(\gamma^{2}-1\right)+1\right]^{-1 / 2}-(\gamma-1)=0 .
$$

A short calculation yields

$$
\left(\sum_{k=1}^{n} q_{k} x_{k}^{2}\right)^{1 / 2}-\sum_{k=1}^{n} q_{k} x_{k} \leqq \frac{(B-A)^{2}}{4(B+A)} .
$$

Equality holds in (5) if and only if there exists a subsequence $\left(k_{1}, k_{2}, \cdots, k_{p}\right)$ of $(1,2, \cdots, n)$ such that

$$
\sum_{m=1}^{p} q_{k_{m}}=\frac{B+3 A}{4(B+A)}(=\theta), \quad x_{k_{m}}=B(m=1,2, \cdots, p),
$$


and $x_{k}=A$ for every $k$ distinct from all $k_{m}$.

EXAMPLE 2, Let $q_{1}, q_{2}, \cdots, q_{n}, A, B, \gamma, x_{1}, x_{2}, \cdots, x_{n}$ be as in Example 1. By Theorem 1 and by the last Remark,

$$
\sum_{k=1}^{n} q_{k} x_{k}-\left(\sum_{k=1}^{n} q_{k} / x_{k}\right)^{-1} \leqq \theta B+(1-\theta) A-\left[\theta B^{-1}+(1-\theta) A^{-1}\right]^{-1},
$$

where $\theta$ is the unique solution in $(0,1)$ of

$$
\gamma-1+\left(\gamma^{-1}-1\right)\left[x\left(\gamma^{-1}-1\right)+1\right]^{-2}=0 .
$$

Solving for $\theta$ and substituting in the last inequality, one gets

$$
\left(\sum_{k=1}^{n} q_{k} x_{k}\right)-\left(\sum_{k=1}^{n} q_{k} / x_{k}\right)^{-1} \leqq\left(B^{1 / 2}-A^{1 / 2}\right)^{2} .
$$

Equality holds in (6) if and only if there exists a subsequence $\left(k_{1}, k_{2}, \cdots, k_{p}\right)$ of $(1,2, \cdots, n)$ such that $\sum_{m=1}^{p} q_{k_{m}}=\left(1+\gamma^{-1 / 2}\right)^{-1}$, $x_{k_{m}}=B(m=1,2, \cdots, p)$, and $x_{k}=A$ for every $k$ distinct from all $k_{m}$.

(6) can be obtained directly. For $k=1,2, \cdots, n$, we have,

$$
\begin{gathered}
x_{k}-(A+B)+A B x_{k}^{-1}=\left(x_{k}-A\right)\left(x_{k}-B\right) x_{k}^{-1} \leqq 0, \\
q_{k} x_{k} \leqq(A+B) q_{k}-A B q_{k} x_{k}^{-1} .
\end{gathered}
$$

So,

$$
\begin{aligned}
& \left(\sum_{k=1}^{n} q_{k} x_{k}\right)-\left(\sum_{k=1}^{n} q_{k} x_{k}^{-1}\right)^{-1} \\
& \leqq A+B-A B\left(\sum_{k=1}^{n} q_{k} x_{k}^{-1}\right)-\left(\sum_{k=1}^{n} q_{k} x_{k}^{-1}\right)^{-1} \\
& =A+B-\left[\left\{A B \sum_{k=1}^{n} q_{k} x_{k}^{-1}\right\}^{1 / 2}-\left\{\sum_{k=1}^{n} q_{k} x_{k}^{-1}\right\}^{-1 / 2}\right]^{2}-2(A B)^{1 / 2} \\
& \leqq A+B-2(A B)^{1 / 2} \\
& =\left(B^{1 / 2}-A^{1 / 2}\right)^{2} .
\end{aligned}
$$

One can also derive from this proof the necessary and sufficient condition given above for equality in (6). (Compare the method of this proof with Diaz and Metcalf [9, §2, Remark 3].)

7. Let $0<m_{1} \leqq a_{j} \leqq M_{1}, 0<m_{2} \leqq b_{j} \leqq M_{2}, j=1,2, \cdots, n, n \geqq 1$, $m_{1} m_{2}<M_{1} M_{2}$, and let $\xi_{1}, \xi_{2}, \cdots, \xi_{n}$ be real numbers $\neq 0$. Set $q_{j}$ $=a_{j} b_{j} \xi_{j}^{2} / \sum_{k=1}^{n} a_{k} b_{k} \xi_{k}^{2}, x_{j}=a_{j} / b_{j}(j=1,2, \cdots, n)$. Observe that

$$
0<m_{1} / M_{2} \leqq x_{j} \leqq M_{1} / m_{2} \quad(j=1,2, \cdots, n) .
$$


By (6),

$$
\begin{aligned}
{\left[\left(\sum_{j=1}^{n} a_{j}^{2} \xi_{j}^{2}\right) / \sum_{j=1}^{n} a_{j} b_{j} \xi_{j}^{2}\right] } & -\left[\left(\sum_{j=1}^{n} a_{j} b_{j} \xi_{j}^{2}\right) / \sum_{j=1}^{n} b_{j}^{2} \xi_{j}^{2}\right] \\
& \leqq\left[\left(M_{1} / m_{2}\right)^{1 / 2}-\left(m_{1} / M_{2}\right)^{1 / 2}\right]^{2} .
\end{aligned}
$$

One obtains also a necessary and sufficient condition for equality in (7). Since the left hand side of (7) is $\geqq 0$ by Cauchy's inequality, (7) may be considered a "complementary" inequality to Cauchy's. Taking $\xi_{1}=\xi_{2} \cdots \xi_{n}=1$, we obtain

$$
\begin{aligned}
{\left[\left(\sum_{j=1}^{n} a_{j}^{2}\right) / \sum_{j=1}^{n} a_{j} b_{j}\right] } & -\left[\left(\sum_{j=1}^{n} a_{j} b_{j}\right) / \sum_{j=1}^{n} b_{j}^{2}\right] \\
& \leqq\left[\left(M_{1} / m_{2}\right)^{1 / 2}-\left(m_{1} / M_{2}\right)^{1 / 2}\right]^{2}
\end{aligned}
$$

8. We give now a complement to Hölder's inequality.

Theorem 2. Let $p>1, p^{-1}+q^{-1}=1,0<A<B$, and let $a_{1}, a_{2}$, $\cdots, a_{n}, b_{1}, b_{2}, \cdots, b_{n}$ be positive numbers with $A \leqq a_{j}^{1 / a} / b_{j}^{1 / p} \leqq B$ $(j=1,2, \cdots, n)$. Set $\gamma=B / A$. Then

$$
\begin{aligned}
0 & \leqq\left[\left(\sum_{j=1}^{n} a_{j}^{p}\right) / \sum_{j=1}^{n} a_{j} b_{j}\right]^{1 / p}-\left[\left(\sum_{j=1}^{n} a_{j} b_{j}\right) / \sum_{j=1}^{n} b_{j}^{q}\right]^{1 / q} \\
& \leqq\left[\theta B^{p}+(1-\theta) A^{p}\right]^{1 / p}-\left[\theta B^{-q}+(1-\theta) A^{-q}\right]^{-1 / q},
\end{aligned}
$$

where $\theta$ is the unique solution in $(0,1)$ of $q\left(\gamma^{p}-1\right)\left[x\left(\gamma^{p}-1\right)+1\right]^{-1 / q}+p\left(\gamma^{-q}-1\right)\left[x\left(\gamma^{-q}-1\right)+1\right]^{-(1 / q)-1}=0$.

Equality on the right in (8) holds if and only if there exists a subsequence $\left(k_{1}, k_{2}, \cdots, k_{t}\right)$ of $(1,2, \cdots, n)$ such that $\left(\sum_{m=1}^{t} a_{k_{m}} b_{k_{m}}\right) / \sum_{j=1}^{n} a_{j} b_{j}=\theta$, $a_{\mathfrak{k}_{m}}^{1 / q} / b_{k_{m}}^{1 / p}=B(m=1,2, \cdots, t)$, and $a_{\mathfrak{k}}^{1 / q} / b_{\mathfrak{k}}^{1 / p}=A$ for every $k$ distinct from all $k_{m}$. Equality on the left in (8) holds if and only if all the ratios $a_{j}^{1 / a} / b_{j}^{1 / p}$ are equal.

Indeed, if we take in Theorem $1, r=-q, s=p, q_{j}=a_{j} b_{j} / \sum_{k=1}^{n} a_{k} b_{k}$, $x_{j}=a_{j}^{1 / q} / b_{j}^{1 / p}(j=1,2, \cdots, n)$, we have by $(3)$,

$$
0 \leqq M_{p}\left(x_{1}, x_{2}, \cdots, x_{n}\right)-M_{-q}\left(x_{1}, x_{2}, \cdots, x_{n}\right) \leqq \Delta .
$$

Equality on the left holds if and only if all the $x_{j}$ are equal. The difference in (9) equals the middle member of (8). The number $\Delta$, by (4) and by the Remark in $\$ 5$, is the right hand member of (8). The necessary and sufficient condition in Theorem 2 for equality on the right in (8) follows, too, from Theorem 1 . The inequality on the left in (8) is, of course, just Hölder's inequality, and the condition given 
for equality there, is just the familiar condition for equality in Hölder's inequality.

9. A number of matrix inequalities follow from Theorems 1 and 2. For these inequalities the reader is referred to [15]. For corresponding Hilbert space in inequalities, see [14].

\section{REFERENCES}

1. E. F. Beckenbach, On the inequality of Kantorovich, Amer. Math. Monthly 71 (1964), 606-619.

2. - A "workshop" on Minkowski's inequality, Inequalities: Proceedings of a Symposium, O. Shisha, ed., Academic Press, New York, 1967.

3. E. F. Beckenbach and R. Bellman, Inequalities, 2nd rev. printing, SpringerVerlag, New York, 1965.

4. G. T. Cargo and O. Shisha, Bounds on ratios of means, J. Res. Nat. Bur. Standards 66B (1962), 169-170.

5. J. B. Diaz, A. J. Goldman and F. T. Metcalf, Equivalence of certain inequalities complementing those of Cauchy-Schwarz and Hölder, J. Res. Nat. Bur. Standards 68B (1964), 147-149.

6. J. B. Diaz and F. T. Metcalf, Stronger forms of a class of inequalities of G. PolyaG. Szeg̈̈, and L. V. Kantorovich, Bull. Amer. Math. Soc. 69 (1963), 415-418.

7. - Complementary inequalities I: Inequalities complementary to Cauchy's inequality for sums of real number, J. Math. Anal. Appl. 9 (1964), 59-74.

8. - Inequalities complementary to Cauchy's inequality for sums of real numbers, Inequalities: Proceedings of a Symposium, O. Shisha, ed., Academic Press, New York, 1967.

9. - Complementary inequalities III: Inequalities complementary to Schwarz's inequality in Hilbert space, Math. Ann. 162 (1965), 120-139.

10. A. J. Goldman, $A$ generalization of Rennie's inequality, J. Res. Nat. Bur. Standards 68B (1964), 59-63.

11. G. H. Hardy, J. E. Littlewood and G. Pólya, Inequalities, 2nd ed., Cambridge Univ. Press, Cambridge, 1952.

12. A. W. Marshall and I. Olkin, Reversal of the Lyapunov, Hölder, and Minkowski inequalities and other extensions of the Kantorovich inequality, J. Math. Anal. Appl. 8 (1964), 503-514.

13. B. Mond and O. Shisha, Ratios of means and applications, Inequalities: Proceedings of a Symposium, O. Shisha, ed., Academic Press, New York, 1967.

14. B. Mond and O. Shisha, $A$ difference inequality for operators in Hilbert space, Blanch Anniversary Volume, B.Mond, ed., Aerospace Research Laboratories, Wright-Patterson Air Force Base, Ohio, 1967.

15. O. Shisha and B. Mond, Bounds on differences of means, Inequalities: Proceedings of a Symposium, O. Shisha, ed., Academic Press, New York, 1967.

Aerospace Research Laboratories

Wright-Patterson AFB, OHIo 